$63^{\text {ème }}$ Congrès de la SFCO, 02009 (2015)

DOI:10.1051/sfco/20156302009

(C) Owned by the authors, published by EDP Sciences, 2015

COMMUNICATION

\title{
Critères d'éligibilité à la réhabilitation implantaire de patients ayant été traités pour un cancer des voies aéro-digestives supérieures : revue de la littérature
}

\author{
Hascoët E*, Valette G**, Le Toux G*, Boisramé S* \\ * Département de chirurgie orale, UFR odontologie-CHRU Brest \\ ** Service d'ORL, CHRU Brest
}

La prise en charge des cancers des voies aéro-digestives supérieures (VADS) peut entraîner des modifications buccales anatomiques et physiologiques (Schiegnitz et al. 2013). Ces dysfonctions altèrent la mastication, la déglutition et la phonation. Suite aux traitements oncologiques (chirurgie, radiothérapie ou radiochimiothérapie), une restauration de ces fonctions, par une réhabilitation dentaire, est nécessaire (Bodard et al. 2006). Les changements de l'anatomie buccale tridimensionnelle induits par la chirurgie, en association ou non avec la radiothérapie, limite la possibilité d'une restauration amovible muco-portée (Korfage et al. 2014). Une forme de crête atypique, des brides cicatricielles et de nouveaux jeux musculaires empêchent la stabilité de cette dernière. La radiothérapie engendre une hyposialie limitant l'adhésion de la prothèse et une hypovascularisation fragilisant la muqueuse. De ce fait, la prothèse implanto-portée semble être la meilleure solution pour réhabiliter ces patients. L'utilisation des implants en territoire irradié a été proposée en 1985 (Jacobsson et al. 1985) et montre des taux de succès élevés, allant de 72 à $100 \%$ (Schiegnitz et al. 2013). Cependant, il n'y a pas à ce jour, de protocole implantaire défini quant à la gestion des conséquences des thérapeutiques anticancéreuses. Les effets de la radiothérapie dépendent de la dose reçue. Du fait de la vascularisation terminale de la mandibule, il est encore difficile de prédire l'impact de l'irradiation sur l'ostéointégration et les risques d'ostéonécrose selon la zone implantaire. Le lambeau libre de péroné présente l'inconvénient d'une absence de vestibule et de gencive attachée kératinisée (Mark et al. 1999). Dans tout les cas, une notion de temps pour l'implantation reste à définir.

L'objectif de cette présentation est de définir des critères d'éligibilité, pour une prise en charge implantaire optimale, chez des patients traités par chirurgie et/ou radiothérapie suite à des cancers des VADS. Ainsi les complications seraient mieux anticipées et pourraient être évitées en modulant la prise en charge. A terme, la définition de critères solides permettrait d'orienter les chirurgies de reconstruction. L'étude doit se baser sur une vision tridimensionnelle et anatomo-fonctionnelle de la cavité orale, en prenant en compte les effets du traitement oncologique (Mark et al. 1999), le pronostic de la maladie et l'implication du patient dans cette restauration.

Nom et adresse du conférencier

Emilie HASCOËT

Département de Chirurgie Orale, UFR odontologie-CHRU Brest

22, avenue Camille Desmoulins C.S. 93837

29238 Brest Cedex 3 (France)

emilie.hascoet1@gmail.com

This is an Open Access article distributed under the terms of the Creative Commons Attribution License 4.0, which permits unrestricted use, distribution, and reproduction in any medium, provided the original work is properly cited. 